\title{
miR-28-5p suppresses cell proliferation and weakens the progression of polycystic ovary syndrome by targeting prokineticin-1
}

\author{
LYUHE MENG $^{1,2}$, HAIYAN YANG ${ }^{2}$, CONGCONG JIN $^{2}$ and SONG QUAN ${ }^{1}$ \\ ${ }^{1}$ Center for Reproductive Medicine, Department of Obstetrics and Gynecology, Nanfang Hospital, \\ Southern Medical University, Guangzhou, Guangdong 510000; ${ }^{2}$ Reproductive Medicine Center, \\ The First Affiliated Hospital of Wenzhou Medical University, Wenzhou, Zhejiang 325000, P.R. China
}

Received October 13, 2018; Accepted April 30, 2019

DOI: $10.3892 / \mathrm{mmr} .2019 .10446$

\begin{abstract}
Prokineticin-1 (PROK1) serves important roles in the pathogenesis of polycystic ovary syndrome (PCOS); however, the association between microRNA (miR)-28-5p and PROK1 remains unclear. In the present study, the roles of miR-28-5p and PROK1, and their interaction in PCOS were investigated. Rat ovary granule cells were transfected with miR-28-5p mimics, and PROK1 expression levels were measured by reverse transcription-quantitative PCR and western blotting. A dual-luciferase reporter assay was performed to determine the association between miR-28-5p and PROK1. Additionally, pcDNA-PROK1 was co-transfected into rat ovary granule cells with miR-28-5p mimics. Cell proliferation, apoptosis, cell cycle and the expression of signaling proteins were investigated using Cell Counting Kit-8 assays, 5-ethynyl-2'-deoxyuridine staining, flow cytometry and western blotting, respectively. PROK1 expression was suppressed in rat ovary granule cells by miR-28-5p mimics, but upregulated following transfection with miR-28-5p inhibitors. The dual-luciferase reporter assay revealed that miR-28-5p binds to the 3'-untranslated region of PROK1. Proliferation activity was increased in PROK1-overexpressing cells; this effect was eliminated by co-transfection with miR-28-5p mimics. PROK1-overexpressing rat ovary granule cells exhibited significantly suppressed cell apoptosis and a decreased number of cells in G1; miR-28-5p mimics reversed these effects. Western blotting revealed that the PI3K/AKT/mTOR signaling pathway was activated by PROK1. The present results suggested that miR-28-5p attenuated the progression of
\end{abstract}

Correspondence to: Professor Song Quan, Center for Reproductive Medicine, Department of Obstetrics and Gynecology, Nanfang Hospital, Southern Medical University, 1023-1063 South Shatai Road, Baiyun, Guangzhou, Guangdong 510000, P.R. China E-mail: quansong@smu.edu.cn

Key words: polycystic ovary microRNA-28-5p, cell proliferation
PCOS by targeting PROK1, which may promote the pathogenesis of PCOS via the PI3K/AKT/mTOR pathway, indicating that the miR-28-5p/PROK1 axis may be a potential therapeutic target for patients with PCOS.

\section{Introduction}

Polycystic ovary syndrome (PCOS) exhibits high morbidity and affects $\sim 4 \%$ of women of reproductive age worldwide $(1,2)$. PCOS is frequently accompanied by a number of alterations in the reproductive, endocrine and metabolic pathways. Patients with PCOS typically present with polycystic ovaries, insulin resistance, chronic anovulation and infertility $(3,4)$. Previous studies revealed that PCOS pathogenesis is due to environmental and genetic factors (5); however, efforts to understand the underlying pathogenic mechanisms are complicated by the heterogeneity of PCOS.

Prokineticin 1 (PROK1), also known as endocrine gland-derived vascular endothelial growth factor (EG-VEGF), is involved in ovarian physiology, endometrial receptivity, embryo implantation and successful pregnancies, together with its receptors [PROK1 receptors (PROKRs)] $(6,7)$. Previous research revealed that PROK1 is an angiogenic and proliferative factor predominantly expressed in the organs of the female reproductive system (8). PROK1 is also involved in female reproductive pathophysiological processes (8). In addition, PROK1 was reported to be differentially expressed during the cycle of vascular morphogenesis in the primate ovary and polycystic human ovaries, compared with VEGF (9-11). There is also evidence that PROK1 mediates placental angiogenesis directly or indirectly (11); however, the precise mechanisms of PROK1 in PCOS require further investigation.

MicroRNAs (miRNAs/miRs) are widely expressed small RNAs that negatively regulate the post-transcriptional expression of functional genes (12). Aberrant expression of miRNAs has been broadly identified as an important factor in the develsyndrome, prokineticin 1, opment of metabolic disorders, such as insulin resistance and obesity $(13,14)$. A recent study reported that miRNAs serve important roles in PCOS, and that the concentration of miR-155 in serum may be a biomarker for monitoring estroprogestinic 
treatment (15). Dysregulated expression of another miRNA, miR-28-5p, has been frequently reported in the development of various types of cancers, including hepatocellular carcinoma, colorectal and prostate cancer (16-18), and most recently ovarian cancer (19). Shi and Teng (18) demonstrated that miR-28-5p expression was downregulated in hepatocellular carcinoma, and that cell proliferation and tube formation capacity were attenuated in cells expressing miR-28-5p. Our previous research indicated that hypermethylation of the miR-28-5p promoter reduces miR-28-5p expression and promotes cell proliferation in rat ovary granulosa cells (Meng et al, unpublished data); however, the downstream mechanisms underlying the effects of miR-28-5p remain unclear.

A previous study indicated that cell proliferation and follicular growth are activated during all stages of ovarian development in PCOS, indicating that cell death in the ovary may mediate follicular atresia (20). There is also evidence that granulosa cell death occurs following oocyte apoptosis (21). Therefore, cell proliferation and survival may be responsible for the development of PCOS.

In the present study, decreased expression of PROK1 was reported in cells transfected with miR-28-5p mimics. A dual-luciferase reporter assay indicated that miR-28-5p targeted the 3'-untranslated region (3'-UTR) of PROK1, attenuating PROK1 expression. Finally, cell proliferation, apoptosis and cell cycle distribution were evaluated following transfection with a PROK1-overexpressing plasmid, in the presence or absence of miR-28-5p mimics. The results of the present study indicated that miR-28-5p inhibited the progression of PCOS by targeting PROK1, which acts via the PI3K/AKT/mTOR signaling pathway, suggesting that PROK1 may be a potentially useful target in the treatment of PCOS.

\section{Materials and methods}

Cell culture and transfection. The rat ovary granulosa cells used in the present study were primary cells prepared by Procell Life Science \& Technology Co., Ltd. (cat. no. CP-R050) via mechanical separation, collagenase digestion and differential adhesion. The cells were cultured in DMEM/F12 (HyClone; GE Healthcare Life Sciences) with $15 \%$ fetal bovine serum (Gibco; Thermo Fisher Scientific, Inc.), epidermal growth factor (10 ng/ml; cat. no. E5036; Sigma-Aldrich; Merck KGaA), and penicillin and streptomycin $(100 \mathrm{U} / \mathrm{ml})$ at $37^{\circ} \mathrm{C}$ and $5 \%$ $\mathrm{CO}_{2}$. miR-28-5p mimics (50 $\left.\mathrm{nM}\right)$, inhibitor (50 $\left.\mathrm{nM}\right)$ and $\mathrm{NC}$ (50 nM) were synthesized by Shanghai GenePharma Co., Ltd., and pcDNA3.0-PROK1 plasmids $(4 \mu \mathrm{g})$ were transfected into cells using Lipofectamine ${ }^{\circledR} 2000$ transfection reagent (Thermo Fisher Scientific, Inc.) according the manufacturer's protocols. Briefly, cells ( $1.5 \times 10^{5}$ cells/well) were seeded in six-well plates containing complete medium without antibiotics for $24 \mathrm{~h}$ prior to transfection. Cell transfection was conducted in six-well plates, in which each well contains a total of $2 \mathrm{ml}$ medium with $5 \mu$ l Lipofectamine 2000 (Invitrogen; Thermo Fisher Scientific, Inc.) and $10 \mu \mathrm{l}$ mimics. Assays were performed $24 \mathrm{~h}$ following cell transfection. Empty pcDNA3.0 vector was used as a negative control (NC) for PROK1 overexpression. Nonsense sequences were used as the NC for miR-28-5p mimics and inhibitors. The sequences of mimics, inhibitor and NC are the as follows: miR-28-5p mimics, 5'-AAGGAGCTC
ACAGTCTATTGAG-3'; miR-28-5p inhibitor 5'-UUCCUC GAGUGUCAGAUAACUC-3'; NC, 5'-UUCUCCGAACGU GUCACGUTT-3'.

Animals. Female Sprague Dawley rats (age, 21 days; weight, $50 \pm 10 \mathrm{~g} ; \mathrm{n}=12$ ) had free access to food and water under a controlled temperature of $23 \pm 2^{\circ} \mathrm{C}$ and humidity (55-65\%) with a 12-h light/dark cycle. A PCOS model was established as previously described (22).

Plasmid construction. Total RNA was extracted using TRIzol reagent (Takara Bio, Inc.) from ovary granulosa cells (cat. no. CP-R050; Procell Life Science \& Technology Co., Ltd.) according to the manufacturer's protocols. Reverse transcription reaction was performed using a Bester ${ }^{\mathrm{TM}}$ qPCR RT Kit (cat. no. DBI-2220; DBI Bioscience). The reverse transcription protocol was as follows: RNA denaturation at $65^{\circ} \mathrm{C}$ for $5 \mathrm{~min}$; genomic DNA removal at $37^{\circ} \mathrm{C}$ for $5 \mathrm{~min}$; reverse transcription at $37^{\circ} \mathrm{C}$ for $15 \mathrm{~min}$; reverse transcriptase inactivation at $98^{\circ} \mathrm{C}$ for $5 \mathrm{~min}$. The vector pcDNA3.0 (Guangzhou Vipotion Biotechnology, Co., Ltd.) was used for the overexpression of PROK1. The coding sequence of PROK1 was cloned using the following primers: Forward 5'-CGGGATCCATGAGAGGTG CTGTGCAAGTCT-3' and reverse, 5'-CCGCTCGAGCTA AAAGTTGACATTCTTCAAGTCC-3'. The PCR mixture contained: 0,25 $\mu \mathrm{l}$ PrimeSTARRHS DNA Polymerase (Clontech Laboratories, Inc.), $5 \mu 1$ XX PrimeSTAR buffer (Clontech Laboratories, Inc.), $2 \mu 1 \mathrm{dNTP}$ Mix (Clontech Laboratories, Inc.); $0.5 \mu 1$ primers (Sangon Biotech Co., Ltd.), $1 \mu \mathrm{l}$ cDNA and $15.75 \mu \mathrm{lddH} 2 \mathrm{O}$, (Takara Bio, Inc.). The thermocycling conditions as follows: Initial denaturation at $94^{\circ} \mathrm{C}$ for $5 \mathrm{~min}$, followed by 30 cycles of $94^{\circ} \mathrm{C}$ for $30 \mathrm{sec} ; 58^{\circ} \mathrm{C}$ for $30 \mathrm{sec} ; 72^{\circ} \mathrm{C}$ for $30 \mathrm{sec} ; 72^{\circ} \mathrm{C} 5 \mathrm{~min}$. Then, amplification products were ligated into the vector using $\mathrm{BamHI}$ and $\mathrm{XhoI}$ restriction sites and identified via double enzyme digestion. Empty vector was used as a NC. Animal experiments were performed in compliance with the ARRIVE guidelines (23), once ethical approval from the Nanfang Hospital Animal Ethics Committee had been obtained.

$R N A$ extraction and reverse transcription-quantitative $P C R$ $(R T-q P C R)$. Total RNAs were extracted using TRIzol ${ }^{\circledR}$ reagent (Takara Bio, Inc.) according to the manufacturer's protocols. Total RNA ( 400 ng) was reverse transcribed into cDNA using a Bestar qPCR RT kit (cat. no. 2200; Kay Biological Technology Co., Ltd.). Samples were incubated at $37^{\circ} \mathrm{C}$ for $15 \mathrm{~min}$ and at $85^{\circ} \mathrm{C}$ for $5 \mathrm{~min}$. U6 small nuclear RNA was used as an internal reference for miR-28-5p expression; primers were obtained from Takara Bio, Inc. GAPDH was used as internal reference; primers were synthesized by Sangon Biotech Co., Ltd. A SYBR Green qPCR kit (Kay Biological Technology Co., Ltd.) was used to quantify miRNA or mRNA expression using a Stratagene Real time PCR system (Mx3000P; Agilent Technologies, Inc.). The amplification reactions were performed under the following conditions: Initial denaturation at $95^{\circ} \mathrm{C}$ for $5 \mathrm{~min}$, followed by 40 cycles of $95^{\circ} \mathrm{C}$ for $15 \mathrm{sec}, 58^{\circ} \mathrm{C}$ for $20 \mathrm{sec}$, and $72^{\circ} \mathrm{C}$ for $30 \mathrm{sec}$. All qPCR reactions were repeated at least three times. Relative mRNA or miRNA expressions were calculated by $2^{-\Delta \Delta \mathrm{Cq}}$ method (24). The primers are listed in Table I. 
Table I. Primer sequences.

\begin{tabular}{ll} 
Gene symbols & \multicolumn{1}{c}{ Sequence (5'-3') } \\
\hline U6 & F: CTCGCTTCGGCAGCACA \\
miR-28-5p & R: AACGCTTCACGAATTTGCGT CTCA \\
Universal reverse & R: ACTGGTGTCGTGGA \\
GAPDH & F: CCTCGTCTCATAGACAAGATGGT \\
& R: GGGTAGAGTCATACTGGAACATG \\
PROK1 & F: CAACTGTCTCTGACTGTGCG \\
& R: AAGGGATCTTGTGGCTTCCA
\end{tabular}

F, forward; R, reverse; PROK1, prokineticin 1; miR, microRNA.

Western blotting. Cells $\left(\sim 10^{6}\right)$ were lysed using protein lysis buffer (cat. no. P0013B; Beyotime Institute of Biotechnology) and total protein was then extracted. Subsequently, a Pierce BCA Protein Assay kit (cat. no. 23227; Pierce; Thermo Fisher Scientific, Inc.) was used to determine the total protein concentration. Equal amounts of total protein $(10 \mu \mathrm{g})$ were separated by $12 \%$ SDS-PAGE. The PVDF membrane was placed in a $5 \%$ BSA blocking solution for $1.5 \mathrm{~h}$ at room temperature. The membranes were incubated at $4^{\circ} \mathrm{C}$ for $12 \mathrm{~h}$ with primary antibodies against PROK1 (1:500; cat. no. ab72807; Abcam), Bcl-2 (1:1,000; cat. no. ab196495; Abcam), Bax (1:1,000; cat. no. ab32503; Abcam), caspase-3 (1:500; cat. no. ab13847; Abcam), p62 (1:1,000; cat. no. 16177; Cell Signaling Technology), cyclin D1 (1:200; cat. no. ab16663; Abcam), PI3K (1:1,000; cat. no. 4255S; Cell Signaling Technology), phosphorylated (p-)PI3K (1:1,000; cat. no. 4228T; Cell Signaling Technology), AKT (1:1,000; cat. no. 4691S; Cell Signaling Technology), p-AKT (1:2000; cat. no. 4060S; Cell Signaling Technology), mTOR (1:1,000; cat. no. 2983S; Cell Signaling Technology), p-mTOR (1:1,000; cat. no. 5536S; Cell Signaling Technology) and GAPDH (1:1,000; cat. no. ab8245; Abcam). The PVDF membrane was placed in a diluted horseradish peroxidase-conjugated rabbit anti-mouse immunoglobulin G secondary antibody (1:10,000; cat. no. ab6728; Abcam) solution and incubated for $1 \mathrm{~h}$ at room temperature. The intensities of labeled proteins were visualized using X-film (cat. no. 4741023951; Yestar Healthcare Holdings Co., Ltd.; http://www.yestarcorp.com/) with an ECL chemiluminescence detection kit (BeyoECL Plus; cat. no. P0018M; Beyotime Institute of Biotechnology) and ImageJ (version 1.8.0; National Institutes of Health) was used to quantify expression by densitometry.

Cell Counting Kit-8 (CCK-8). Cells $\left(2 \times 10^{4}\right)$ transfected with exogenous sequences were suspended in $200 \mu \mathrm{l}$ culture medium and seeded into 96-well plates. The CCK-8 assay was conducted following culture under standard conditions $\left(37^{\circ} \mathrm{C}\right.$ ) for a further $48 \mathrm{~h}$. Then, $10 \mu \mathrm{l} \mathrm{CCK}-8$ reagent (Beijing Solarbio Science \& Technology Co., Ltd.) was added to each well, followed by incubation for a further $1 \mathrm{~h}$ at $37^{\circ} \mathrm{C}$. Finally, the absorbance at $450 \mathrm{~nm}$ was detected for quantification of cell proliferation rates using a plate reader (BioTek Instruments, Inc.).
5-Ethynyl-2'-deoxyuridine (EdU) staining and flow cytometry. Cells $\left(10^{4}\right)$ transfected with exogenous sequences were plated into 96-well plates and cultured for $48 \mathrm{~h}$. Then, $100 \mu \mathrm{l}$ EdU reagent (cat. no. B23151; Invitrogen; Thermo Fisher Scientific, Inc.) was added to each well after washing with PBS. The cells were incubated at $37^{\circ} \mathrm{C}$ for $2 \mathrm{~h} .100 \mu \mathrm{l}$ glycerol was added to each well for neutralization after cells were fixed with $4 \%$ paraformaldehyde for $15 \mathrm{~min}$ at room temperature. Subsequently, $0.5 \%$ Triton X-100 was added into each well, and cells were incubated for $10 \mathrm{~min}$ at room temperature, followed by washing with PBS. Apollo reagents and Hoechst 33342 staining reagent included in an EdU kit (cat. no. CA1170; Beijing Solarbio Science \& Technology Co., Ltd.) were used according to the manufacturer protocols. Then, the cells were incubated for $30 \mathrm{~min}$ at room temperature. Cells were analyzed via flow cytometry and the software used for the analysis was Cytomics FC500 Flow Cytometry CXP Software v2.0 (CytoFLEX; Beckman Coulter, Inc.).

Cell apoptosis and cell cycle distribution. Following transfection, $10^{4}$ cells were cultured for $48 \mathrm{~h}$, and the apoptosis of cells was then analyzed using the Annexin V-FITC/propidium iodide (PI) cell apoptosis detection kit (BD Biosciences) containing according to the manufacturer's protocols, and cells were incubated at $4^{\circ} \mathrm{C}$ for $30 \mathrm{~min}$. Cells with positive signals for Annexin V-FITC and PI were in the late stage of apoptosis, whereas cells with positive signals for Annexin V-FITC only were in the early apoptosis stage. The total apoptosis ratio was defined by the summation of late-apoptotic and early-apoptotic cells.

Following transfection of cells with exogenous sequences, cells were stained with PI dye was incubated at $4^{\circ} \mathrm{C}$ for $30 \mathrm{~min}$ to determine the cell cycle distribution. A flow cytometer was used to analyze the cells and the software used for the analysis was Cytomics FC500 Flow Cytometry CXP Software v2.0 (Beckman Coulter, Inc.).

Dual-luciferase reporter assay. TargetScan 7.2 (http://www. targetscan.org) was used to identify the putative targets of miR-28-5p, and PROK1 was found to be one of the targets of miR-28-5p. The software was used according to previous report (25). The 3'-UTR sequences of the PROK1 gene were obtained using primers (forward, 5'-CCGCTCGAGTGGGTG ACCTCTTGTGTTACATCTG-3' and reverse, 5'-ATTTGC GGCCGCCCCAGCCCACCTCACACTCATA-3'; Sangon Biotech Co., Ltd.). Mutated (MUT) 3'-UTR sequences were amplified using paired primers with a mismatched nucleotide introduced in the upstream primer (MUT; forward, 5'-TTTCTT TCATTGGCTCCGATGATGTTTTAGGAGTAAG-3' and reverse, 5'-CTTACTCCTAAAACATCATCGGAGCCAATG AAAGAAA-3'; Sangon Biotech Co., Ltd.). Amplification products including the wild-type (WT) and MUT 3'-UTRs of PROK1 were then separately ligated into Psi-CHECK2 plasmids (cat. no. C8021; Promega Corporation). Psi-CHECK2 plasmids were co-transfected with miR-28-5p mimics or NC into cells using Lipofectamine $2000^{\mathrm{TM}}$ and incubated for $48 \mathrm{~h}$ at $37^{\circ} \mathrm{C}$. The luciferase activities of firefly and Renilla were measured with the Dual-Luciferase Reporter Assay system (cat. no. C8021; Promega Corporation) according to the manufacturer's protocol. Firefly luciferase activity was normalized 
to Renilla luciferase activity. Cell lysates were collected using a GloMax machine (Promega Corporation).

Statistical analysis. Data from $>3$ biological replicates for each assay were presented as the mean \pm standard deviation, and data were analyzed using SPSS 16.0 software (SPSS, Inc.). Student's t-test was used to analyze differences between two groups, and one-way analysis of variance followed by Tukey's test was performed to compare differences between three or more groups.

\section{Results}

miR-28-5p suppresses PROK1 expression by targeting its 3 '-UTR. To investigate the relationship between miR-28-5p and PROK1, the mRNA expression levels of PROK1 were analyzed by qPCR, and the protein expression was evaluated via western blotting following transfection of ovary granule cells with miR-28-5p mimics. It was demonstrated that mRNA expression of PROK1 was significantly suppressed in miR-28-5p overexpressing cells compared with the NC (Fig. 1A). Similarly, the protein expression of PROK1 was significantly downregulated following the transfection of ovary granule cells with miR-28-5p mimics compared with the NC (Fig. 1B). Conversely, transfection with miR-28-5p inhibitor induced the opposite results in PROK1 expression in ovary granule cells (Fig. 1A and B).

To determine whether miR-28-5p binds to PROK1, the 3'-UTR of PROK1 was cloned and inserted into psi-CHECK2 plasmids. For comparison, the binding site predicted by the online database TargetScan was mutated, and then also inserted into psi-CHECK2 plasmids (Fig. 1C). Cells co-transfected with WT 3'-UTR and miR-28-5p exhibited significantly reduced luciferase activity compared with cells transfected with NC or MUT 3'-UTR (Fig. 1D). The results indicated that miR-28-5p negatively regulated PROK1 expression via direct binding to its 3 '-UTR.

PCOS development promoted by PROK1 overexpression is inhibited by miR-28-5p via the PI3K/AKT/mTOR signaling pathway. PCOS is a common endocrine-reproductive disease in females and it has been demonstrated that ovary granular cells in PCOS patients showed increased apoptotic rates $(17,26)$. To verify whether PROK1 promotes PCOS in rat ovary granule cells, a PROK1 overexpression plasmid was constructed. Additionally, a rescue experiment series was designed to further demonstrate the influence of the interaction between miR-28-5p and PROK1 on rat ovary granule cell survival. As presented in Fig. 2A, a western blot assay verified the overexpression of the PROK1 plasmid, revealing that PROK1 was significantly upregulated in cells transfected with the PROK1 overexpression plasmid compared with empty vector. The overexpression of PROK1 protein was accompanied by significantly increased cell proliferation rates in rat ovary granule cells (Fig. 2B and C); however, the effects of PROK1 upregulation on proliferation were attenuated by co-expression of PROK1 and miR-28-5p mimics, compared with cells transfected with pcDNA-PROK1 alone.

Additionally, apoptosis was significantly suppressed in rat ovary granule cells overexpressing PROK1 compared with

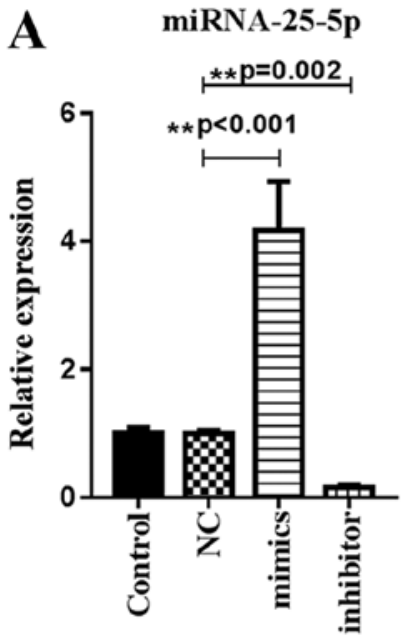

PROK1
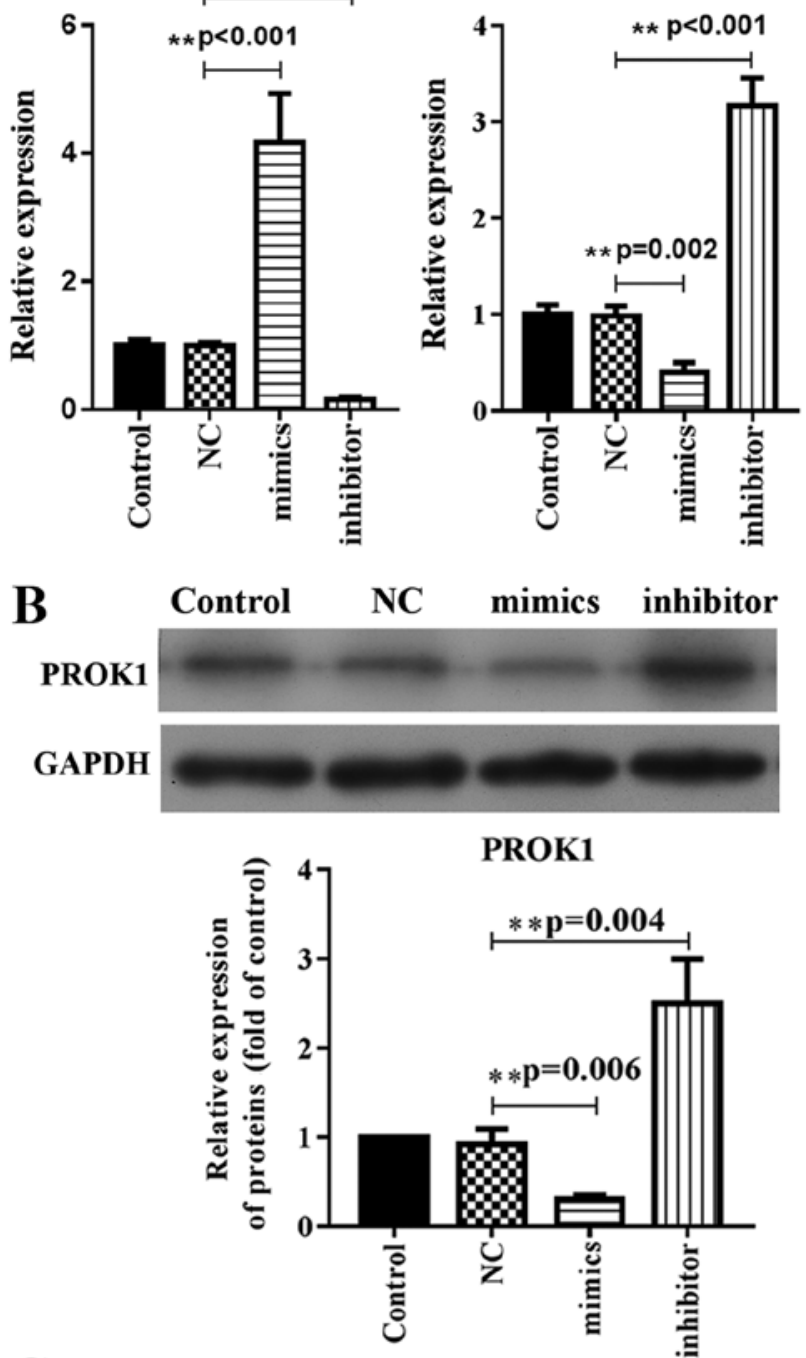

C

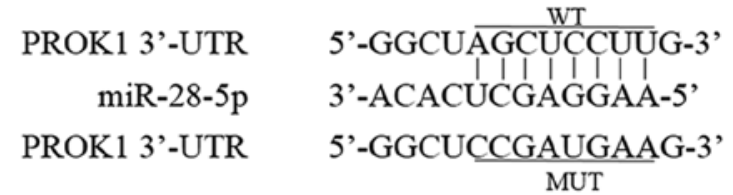

D

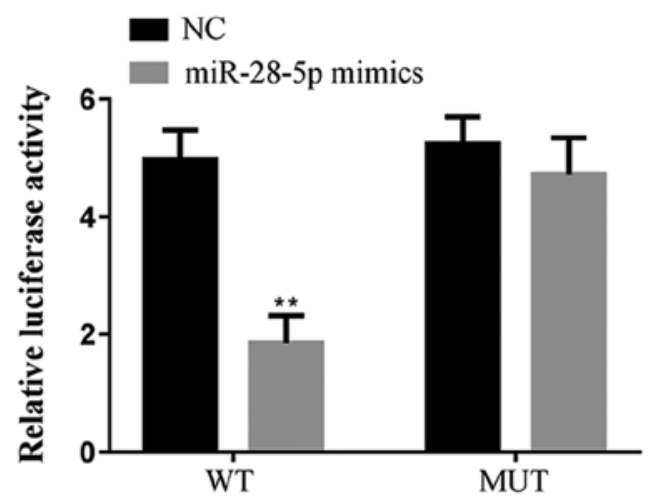

Figure 1. miR-28-5p suppresses PROK1 expression by targeting the 3'-UTR of PROK1. (A) mRNA expression of miR-28-5p and PROK1 in rat ovary granule cells. (B) Protein expression of PROK1. (C) WT and MUT sequences in the 3'-UTR of PROK1 involved in binding with miR-28-5p. ${ }^{* *} \mathrm{P}<0.01$, as indicated. (D) Dual-luciferase reporter assay confirming an interaction between miR-28-5p and the PROK1 3'-UTR. All data are presented as the mean $\pm \mathrm{SD} .{ }^{* *} \mathrm{P}<0.01$ vs. $\mathrm{NC}(\mathrm{n}=3)$. miR-28-5p, microRNA-28-5p; PROK1, prokineticin 1; 3'-UTR, 3'-untranslated region; NC, negative control; WT, wild type; MUT, mutant. 
A
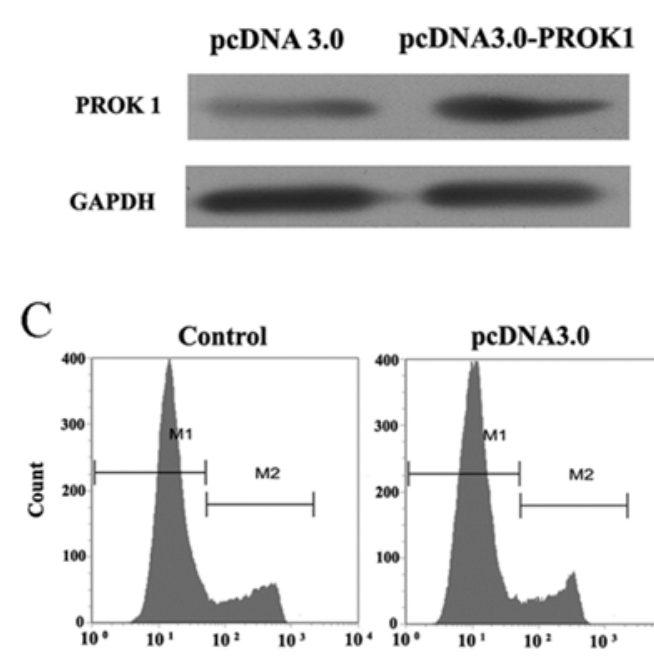

pcDNA3.0

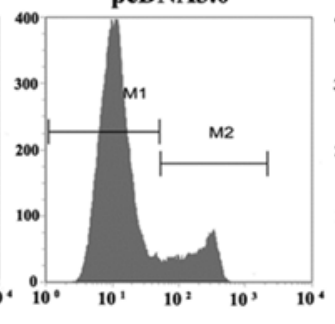

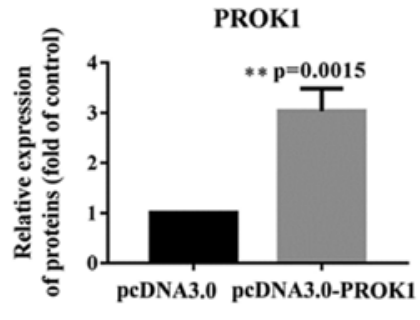

\section{B}

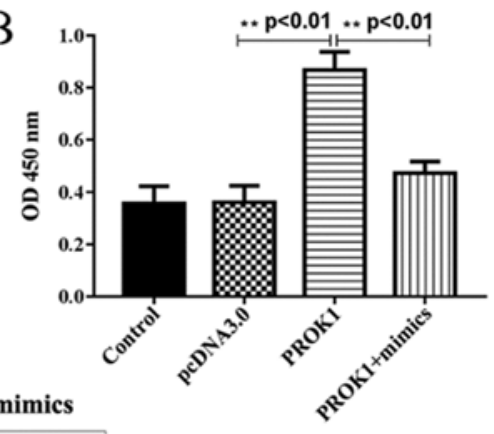

PROK1

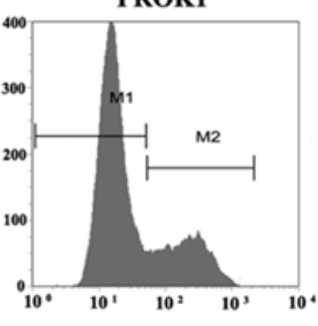

PROK1+mimics

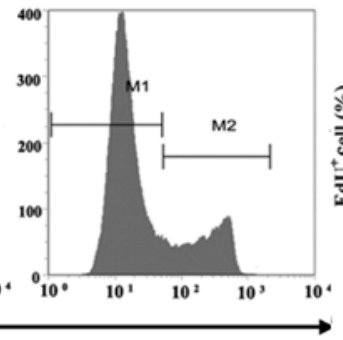

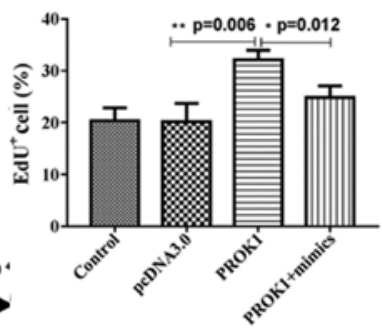

Channels (FL2-H)

Figure 2. miR-28-5p attenuates the PROK1-mediated proliferation of rat ovary granulosa cells. (A) Western blot analysis of PROK1 protein expression following transfection with pcDNA3.0 PROK1. ${ }^{* *} \mathrm{P}=0.0015$ vs. pcDNA3.0. (B) Cell Counting Kit-8 assay demonstrating that miR-28-5p inhibited the PROK1-promoted proliferation of rat ovary granulosa cells. (C) Enhanced proliferation following overexpression of PROK1 was reversed by miR-28-5p mimics in rat ovary granulosa cells, as determined by EdU staining. All data are presented as the mean $\pm \mathrm{SD}$. $\mathrm{P}<0.05$ and ${ }^{* * *} \mathrm{P}<0.01$, as indicated $(\mathrm{n}=3)$. miR-28-5p, microRNA-28-5p; PROK1, prokineticin 1; mimics, miR-28-5p mimics; EdU, 5-ethynyl-2'-deoxyuridine.

empty vector (Fig. 3A and B), and a significantly increased percentage of these cells were in S phase (Fig. 3C and D). These tendencies were attenuated by co-transfection with miR-28-5p mimics, which elevated cell apoptosis, and redistributed cells to the G0/G1 phase compared with pcDNA-PROK1 alone. The expression of apoptosis- and cell cycle-associated proteins was investigated via western blotting (Fig. 3E). Compared with empty pcDNA3.0 vector, transfection with pcDNA3-PROK1 significantly upregulated Bcl-2 and cyclin D1 expression, whereas caspase-3, Bax and p62 expression levels were downregulated; however, co-transfection with miR-28-5p mimics attenuated these effects. Additionally, it was revealed via western blotting that $\mathrm{PI} 3 \mathrm{~K} / \mathrm{AKT} / \mathrm{mTOR}$ activation was also potentiated in PROK1-overexpressing cells compared with the control (Fig. 3F). Conversely, this activation was reversed by co-transfection with miR-28-5p mimics. These results indicated that PROK1 promoted the proliferation and suppressed the apoptosis of rat ovary granule cells via the $\mathrm{PI} 3 \mathrm{~K} / \mathrm{AKT} / \mathrm{mTOR}$ signaling pathway.

\section{Discussion}

Abnormal expression of miRNAs has been reported in a variety of cancers, resulting in increased attention regarding the potential pathogenic effects of miRNAs on cancer initiation and progression $(27,28)$. miR-28-5p has been reported to be closely associated with tumor carcinogenesis. For example, hepatocellular carcinoma progression was suppressed by miR-28-5p via the targeting of insulin-like growth factor-1 (IGF-1) (18); miR-28-5p also exerts a number of antitumor effects in renal cell carcinoma via the regulation of Ras-related protein Rap-1B (29). Additionally, miR-28-5p was reported to be downregulated in colorectal cancer tissue compared with in normal colon tissue, and to suppress colorectal cancer cell proliferation, migration and invasion (30). Conversely, certain studies have suggested that miR-28-5p may also serve as a potential marker for the development and progression of cancers $(19,31)$. For example, it was reported that miR-28-5p may promote the development and progression of renal cell carcinoma by increasing the risk of chromosomal instability and promoting checkpoint weakness (31). Additionally, miR-28-5p acted as a carcinogenic biomarker to enhance ovarian cancer cell proliferation, inhibit cell apoptosis, and contribute to migration and invasion (19). In the present study, it was demonstrated that miR-28-5p suppressed cell proliferation, and induced cell cycle arrest and apoptosis in PCOS. Therefore, miR-28-5p regulate PCOS and inhibit PCOS progression.

It has been previously reported that miRNAs contribute to the regulation of various physiological processes by targeting mRNAs $(32,33)$. And the activation or suppression of the mRNA by these miRNAs could induce positive or negative effects on the progression of various diseases, including PCOS (34-36). For example, miR-324-3p expression was observed to be reduced in PCOS; miR-324-3p inhibited the proliferation, and promoted the apoptosis of granulosa cells by targeting Wnt-2B (37). In addition, miR-93 promoted ovarian granulosa cell proliferation by targeting cyclin-dependent kinase inhibitor $1 \mathrm{~A}$ in PCOS (38). miR-483 suppressed cell proliferation in PCOS by targeting IGF-1 (39). In the present study, it was demonstrated that miR-28-5p negatively regulated PROK1 expression by binding to the 3'-UTR of PROK1.

The present findings suggested that miR-28-5p was involved in PCOS by regulating PROK1. PROK1 serves an important role in angiogenesis, and it was observed that the concentration of VEGF in the ovaries of females with PCOS 
A

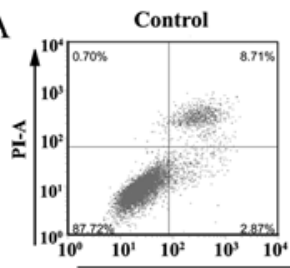

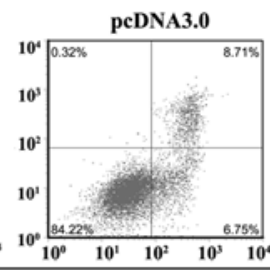
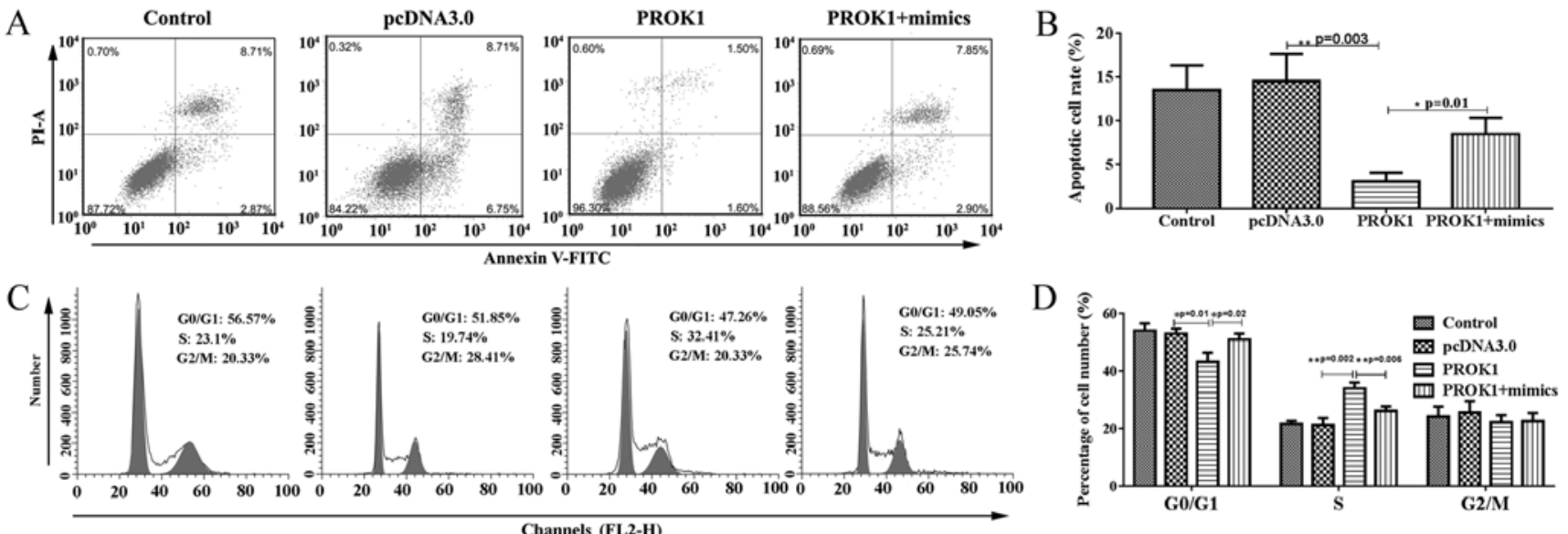

E
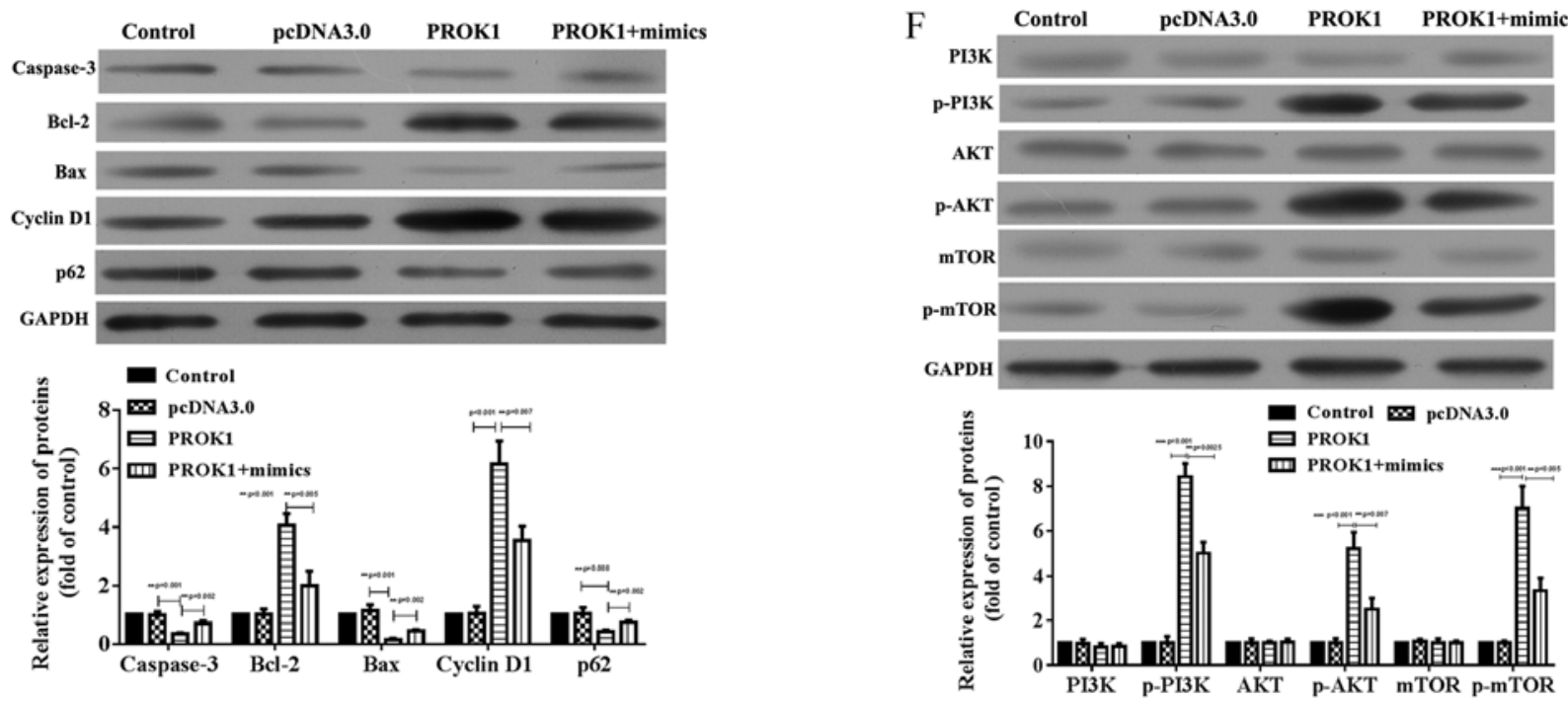

Figure 3. PROK1-induced apoptosis suppression and cell cycle distribution shift are reversed by miR-28-5p mimics in rat ovary granulosa cells. (A and B) Decreased apoptosis in cells co-transfected with miR-28-5p and PROK1 was observed when compared with cells transfected with PROK1 alone, as determined by flow cytometry. (C and D) Cell cycle distribution in cells co-transfected with mimics and PROK1 compared with PROK-transfected cells alone, as determined by flow cytometry. (E) Altered expression of Bax, Bcl-2, Caspase 3, p21 and cyclin D protein induced by PROK1 overexpression were reversed by miR-28-5p. (F) Activation of the PI3K/AKT/mTOR signaling pathway following transfection with pcDNA-PROK1, or co-transfection with pcDNA-PROK1 and mimics. All data are presented as the mean \pm SD. ${ }^{*} \mathrm{P}<0.05,{ }^{* *} \mathrm{P}<0.01$ and ${ }^{* * *} \mathrm{P}<0.001$, as indicated $(\mathrm{n}=3)$. miR-28-5p, microRNA-28-5p; $\mathrm{PROK} 1$, prokineticin 1; mimics, miR-28-5p mimics; $\mathrm{p}-$, phosphorylated; PI, propidium iodide.

was increased compared with healthy females (40). In addition, previous studies also demonstrated that PROK1 promoted human umbilical vein endothelial cell proliferation, differentiation and survival $(7,41)$. Furthermore, PROK1 also promoted the morphogenesis of vascular endothelial cells into tube-like structures, as observed in 2D-model experiments (42). In the present study, it was demonstrated that the overexpression of PROK1 promoted cell proliferation, and inhibited cell cycle arrest and apoptosis in PCOS. The present findings further indicated that PROK1 may accelerate PCOS progression.

The cell cycle is a series of important cellular events that occurs during various physiological and pathological processes, and the initiation of G1 phase is critical during cell cycle progression $(43,44)$. Cyclins are important regulators of cell cycle progression. Cyclin D1, as a key regulatory protein of G1 phase progression, has been established as a major indicator of cell cycle progression, with increased sensitivity when compared with other cyclins (45). Specifically, cyclin D1 promotes the transition from $\mathrm{G} 1$ to $\mathrm{S}$ phase by binding and activating cyclin-dependent kinase 4 (CDK4), which accelerates cell proliferation (46). Previous studies have reported that p62 serves important roles in cell cycle progression by reducing the ubiquitinylation-mediated degradation of CDK1 and accelerating G2/M phase progression $(47,48)$. In the present study, it was demonstrated that PROK1 upregulated cyclin D1 expression and downregulated p62 expression in ovary granule cells, whereas miR-28-5p mimics reversed these effects, which indicated that miR-28-5p promoted cell cycle arrest by targeting PROK1 in PCOS.

Apoptosis is an important biological death process that is genetically controlled, which serves as an important mechanism for maintaining a stable internal environment (49). Caspase-3, Bcl-2 and Bax are central functional proteins in apoptosis pathways (50). Caspases are a class of cysteine aspartic acid-specific proteases in the cytoplasm; caspase-3, also known as $32 \mathrm{kDa}$ cysteine protease, is a member of the interleukin-lp-converting enzyme family (51). Caspase-3 is a common downstream factor in various apoptotic pathways. Bcl-2 exhibits anti-apoptotic effects, whereas Bax protein is structurally similar to Bcl-2 but induces opposing effects on cell apoptosis (52). In the present 
study, PROK1 decreased caspase- 3 and Bax expression, and increased Bcl-2 expression, whereas treatment with miR-28-5p mimics attenuated these alterations in protein expression. Therefore, it was further indicated that miR-28-5p promoted cell apoptosis by targeting PROK1 in PCOS.

The PI3K/AKT pathway serves an important role in mediating ovarian granular cell function (53-56). PI3K activation induces the phosphorylation of AKT proteins and mTOR gene expression, leading to the activation of downstream signaling associated with cell proliferation, survival and angiogenesis (57). Patients with PCOS show decreased ovary granular cells caused by decreasing viability of cell proliferation or survival (20). Previous studies showed that the PI3K/AKT signaling pathway is activated in PCOS $(53,54)$. Upregulation of Wnt-5a, which acts as a proinflammatory factor, is a signal to PI3K/AKT, activating inflammation and oxidative stress in the granulosa cells of patients with PCOS via NF- $\kappa B$ (53). Additionally, Xin et al (55) reported that patients with insulin resistance underwent significant alterations to the PI3K/AKT/GSK3 signaling pathway. The present study demonstrated that PROK1 affect the progression of PCOS involved in the PI3K/AKT/mTOR signaling pathway.

In conclusion, these findings suggested that miR-28-5p could attenuate the progression of PCOS by targeting the 3'-UTR of PROK1which may involved in the PI3K/AKT/mTOR pathway, indicating that the miR-28-5p/PROK1 axis may be a potential therapeutic target for patients with PCOS.

\section{Acknowledgements}

Not applicable.

\section{Funding}

No funding was received.

\section{Availability of data and materials}

The datasets used and/or analyzed during the current study are available from the corresponding author on reasonable request.

\section{Authors' contributions}

LM made substantial contributions to the conception and design of the study. HY contributed to data acquisition, data analysis and data interpretation. CJ performed some experiments, analyzed the data and drafted the article. SQ was involved in the design of the experiments and critically revised the manuscript for important intellectual content in the submission process. All authors agree to be accountable for all aspects of the work in ensuring that questions related to the accuracy or integrity of the work are appropriately investigated and resolved. All authors have read and approved the final manuscript.

\section{Ethics approval and consent to participate}

Animal experiments were approved by the Nanfang Hospital Animal Ethic Committee, and conducted in accordance with the ARRIVE guidelines.

\section{Patient consent for publication}

Not applicable.

\section{Competing interests}

The authors declare that they have no competing interests.

\section{References}

1. Hull MG: Epidemiology of infertility and polycystic ovarian disease: Endocrinological and demographic studies. Gynecol Endocrinol 1: 235-245, 1987.

2. Knochenhauer ES, Key TJ, Kahsar-Miller M, Waggoner W, Boots LR and Azziz R: Prevalence of the polycystic ovary syndrome in unselected black and white women of the southeastern United States: A prospective study. J Clin Endocrinol Metab 83: 3078-3082, 1998.

3. Wajid S and Rashid N: miRNA signatures and their potential role in PCOS. Int J Gen Cancer 1: 28-36, 2015.

4. Eser A, Hizli D, Namuslu M, Haltas H, Kosus N, Kosus A and Kafali H: Protective effect of curcumin on ovarian reserve in a rat ischemia model: An experimental study. Clin Exp Obstet Gynecol 44: 453-457, 2017.

5. Diamanti-Kandarakis E, Piperi C, Spina J, Argyrakopoulou G, Papanastasiou L, Bergiele A and Panidis D: Polycystic ovary syndrome: The influence of environmental and genetic factors. Hormones (Athens) 5: 17-34, 2006.

6. Brouillet S, Hoffmann P, Thomas-Cadi C, Bergues U, Feige JJ, Alfaidy N and Hennebicq S: PROK1, prognostic marker of embryo implantation? Gynecol Obstet Fertil 41: 562-565, 2013 (In French).

7. Lecouter J, Kowalski J, Foster J, Hass P, Zhang Z, Dillard-Telm L, Frantz G, Rangell L, DeGuzman L, Keller GA, et al: Identification of an angiogenic mitogen selective for endocrine gland endothelium. Nature 412: 877-884, 2001.

8. Brouillet S, Hoffmann P, Feige JJ and Alfaidy N: EG-VEGF: A key endocrine factor in placental development. Trends Endocrinol Metab 23: 501-508, 2012.

9. Fraser HM and Duncan WC: Vascular morphogenesis in the primate ovary. Angiogenesis 8: 101-116, 2005.

10. Ferrara N, Frantz G, Lecouter J, Dillard-Telm L, Pham T, Draksharapu A, Giordano T and Peale F: Differential expression of the angiogenic factor genes vascular endothelial growth factor (VEGF) and endocrine gland-derived VEGF in normal and polycystic human ovaries. Am J Pathol 162: 1881-1893, 2003.

11. Alfaidy N, Hoffmann P, Boufettal H, Samouh N, Aboussaouira T, Benharouga M, Feige JJ and Brouillet S: The multiple roles of EG-VEGF/PROK1 in normal and pathological placental angiogenesis. Biomed Res Int 2014: 451906, 2014.

12. Voorhoeve PM, le Sage CL, Schrier M, Gillis AJ, Stoop H, Nagel R, Liu YP, van Duijse J, Drost J, Griekspoor A, et al: A genetic screen implicates miRNA-372 and miRNA-373 as oncogenes in testicular germ cell tumors. Cell 124: 1169-1181, 2006.

13. Takanabe R, Ono K, Abe Y, Takaya T, Horie T, Wada H, Kita T, Satoh N, Shimatsu A and Hasegawa K: Up-regulated expression of microRNA-143 in association with obesity in adipose tissue of mice fed high-fat diet. Biochem Biophys Res Commun 376: 728-732, 2008.

14. Murri M, Insenser M, Fernández-Durán E, San-Millán JL and Escobar-Morreale HF: Effects of polycystic ovary syndrome (PCOS), sex hormones, and obesity on circulating miRNA-21, miRNA-27b, miRNA-103, and miRNA-155 expression. J Clin Endocrinol Metab 98: E1835-E1844, 2013.

15. Arancio W, Calogero Amato M, Magliozzo M, Pizzolanti G, Vesco R and Giordano C: Serum miRNAs in women affected by hyperandrogenic polycystic ovary syndrome: The potential role of miR-155 as a biomarker for monitoring the estroprogestinic treatment. Gynecol Endocrinol 34: 704-708, 2018.

16. Almeida MI, Nicoloso MS, Zeng L, Ivan C, Spizzo R, Gafà R, Xiao L, Zhang X, Vannini I, Fanini F, et al: Strand-specific miR-28-5p and miR-28-3p have distinct effects in colorectal cancer cells. Gastroenterology 142: 886-896, 2012.

17. Rizzo M, Berti G, Russo F, Evangelista M, Pellegrini M and Rainaldi G: The miRNA pull out assay as a method to validate the mir-28-5p targets identified in other tumor contexts in prostate cancer. Int J Genomics 2017: 5214806, 2017. 
18. Shi X and Teng F: Down-regulated miR-28-5p in human hepatocellular carcinoma correlated with tumor proliferation and migration by targeting insulin-like growth factor-1 (IGF-1). Mol Cell Biochem 408: 283-293, 2015.

19. Xu J, Jiang N, Shi H, Zhao S, Yao S and Shen H: miR-28-5p promotes the development and progression of ovarian cancer through inhibition of N4BP1. Int J Oncol, 2017 (Epub ahead of print).

20. Hughesdon PE: Morphology and morphogenesis of the Stein-Leventhal ovary and of so-called 'hyperthecosis'. Obstet Gynecol Surv 37: 59-77, 1982.

21. Morita Y and Tilly JL: Oocyte apoptosis: Like sand through an hourglass. Dev Biol 213: 1-17, 1999.

22. Kim EJ, Jang M, Choi JH, Park KS and Cho IH: An improved dehydroepiandrosterone-induced rat model of polycystic ovary syndrome (PCOS): Post-pubertal improve PCOS's features. Front Endocrinol (Lausanne) 9: 735, 2018.

23. Kilkenny C, Browne WJ, Cuthill IC, Emerson M and Altman DG: Improving bioscience research reporting: The ARRIVE guidelines for reporting animal research. PLoS One 8: 1000412, 2010

24. Schmittgen TD: Real-time quantitative PCR. Methods 25: 383-385, 2001.

25. Agarwal V, Bell GW, Nam JW and Bartel DP: Predicting effective microRNA target sites in mammalian mRNAs. Elife 4: 2015.

26. Ma HX, Xie J and Lai MH: Effects of yangling zhongyu decoction on the secretion of ovarian granule cells in polycystic ovarian syndrome rat model. Zhongguo Zhong Xi Yi Jie He Za Zhi 32: 54-57, 2012 (In Chinese).

27. Chi XX, Zhang T, Chu XL, Zhen JL and Zhang DJ: The regulatory effect of genistein on granulosa cell in ovary of rat with PCOS through Bcl-2 and Bax signaling pathways. J Vet Med Sci 80: 1348-1355, 2018.

28. Tutar Y: miRNA and cancer; Computational and experimental approaches. Curr Pharm Biotechnol 15: 429, 2014.

29. Wang WT and Chen YQ: Circulating miRNAs in cancer: From detection to therapy. J Hematol Oncol 7: 86, 2014

30. Wang C, Wu C, Yang Q, Ding M, Zhong J, Zhang CY, Ge J, Wang $\mathrm{J}$ and Zhang C: miR-28-5p acts as a tumor suppressor in renal cell carcinoma for multiple antitumor effects by targeting RAP1B. Oncotarget 7: 73888-73902, 2016.

31. Hell MP, Thoma CR, Fankhauser N, Christinat Y, Weber TC and Krek W: miR-28-5p promotes chromosomal instability in VHL-associated cancers by inhibiting Mad2 translation. Cancer Res 74: 2432-2443, 2014.

32. Amirkhah R, Schmitz U, Linnebacher M, Wolkenhauer O and Farazmand A: MicroRNA-mRNA interactions in colorectal cancer and their role in tumor progression. Genes Chromosomes Cancer 54: 129-141, 2015.

33. Giza DE, Vasilescu C and Calin GA: MicroRNAs and ceRNAs: Therapeutic implications of RNA networks. Expert Opin Boil Ther 14: 1285-1293, 2014.

34. Devaraj S and Natarajan J: miRNA-mRNA network detects hub mRNAs and cancer specific miRNAs in lung cancer. In Silico Boil 11: 281-295, 2011.

35. Huang X, Liu C, Hao C, Tang Q, Liu R, Lin S, Zhang L and Yan W: Identification of altered microRNAs and mRNAs in the cumulus cells of PCOS patients: MiRNA-509-3p promotes oestradiol secretion by targeting MAP3K8. Reproduction 151: 643-655, 2016.

36. Song J, Luo S and Li SW: miRNA-592 is downregulated and may target LHCGR in polycystic ovary syndrome patients. Reprod Boil 15: 229-237, 2015.

37. Jiang YC and Ma JX: The role of MiR-324-3p in polycystic ovary syndrome (PCOS) via targeting WNT2B. Eur Rev Med Pharmacol Sci 22: 3286-3293, 2018.
38. Jiang L, Huang J, Li L, Chen Y, Chen X, Zhao X and Yang D: MicroRNA-93 promotes ovarian granulosa cells proliferation through targeting CDKN1A in polycystic ovarian syndrome. J Clin Endocrinol Metab 100: E729-E738, 2015.

39. Xiang Y, Song Y, Li Y, Zhao D, Ma L and Tan L: miR-483 is down-regulated in polycystic ovarian syndrome and inhibits KGN cell proliferation via targeting insulin-like growth factor 1 (IGF1). Med Sci Monit 22: 3383-3393, 2016.

40. Agrawal R, Jacobs H, Payne N and Conway G: Concentration of vascular endothelial growth factor released by cultured human luteinized granulosa cells is higher in women with polycystic ovaries than in women with normal ovaries. Fertil Steril 78: 1164-1169, 2002

41. Lin R, Lecouter J, Kowalski J and Ferrara N: Characterization of endocrine gland-derived vascular endothelial growth factor signaling in adrenal cortex capillary endothelial cells. J Biol Chem 277: 8724-8729, 2002.

42. Murray JD: On the mechanochemical theory of biological pattern formation with application to vasculogenesis. C R Biol 326: 239-252, 2003.

43. Bertoli C, Skotheim JM and de Bruin RA: Control of cell cycle transcription during G1 and S phases. Nat Rev Mol Cell Boil 14: 518-528, 2013.

44. Karamysheva Z, Diaz-Martinez LA, Warrington R and Yu H: Graded requirement for the spliceosome in cell cycle progression. Cell Cycle 14: 1873-1883, 2015.

45. Lim S and Kaldis P: Cdks, cyclins and CKIs: Roles beyond cell cycle regulation. Development 140: 3079-3093, 2013.

46. Aleem E and Arceci RJ: Targeting cell cycle regulators in hematologic malignancies. Front Cell Dev Biol 3: 16, 2015.

47. Li H, Peng X, Wang Y, Cao S, Xiong L, Fan J, Wang Y, Zhuang S, $\mathrm{Yu} \mathrm{X}$ and Mao H: Atg5-mediated autophagy deficiency in proximal tubules promotes cell cycle G2/M arrest and renal fibrosis. Autophagy 12: 1472-1486, 2016.

48. Zhang J, Yang Z and Dong J: P62: An emerging oncotarget for osteolytic metastasis. J Bone Oncol 5: 30-37, 2016.

49. Hassan M, Watari H, AbuAlmaaty A, Ohba Y and Sakuragi N: Apoptosis and molecular targeting therapy in cancer. Biomed Res Int 2014: 150845, 2014

50. Kuwana T and Newmeyer DD: Bcl-2-family proteins and the role of mitochondria in apoptosis. Curr Opin Cell Biol 15: 691-699, 2003.

51. Porter AG and Janicke RU: Emerging roles of caspase-3 in apoptosis. Cell Death Differ 6: 99-104, 1999.

52. Breckenridge DG and Xue D: Regulation of mitochondrial membrane permeabilization by BCL-2 family proteins and caspases. Curr Opin Cell Biol 16: 647-652, 2004.

53. Zhao Y, Zhang C, Huang Y, Yu Y, Li R, Li M, Liu N, Liu P and Qiao J: Upregulated expression of WNT5a increases inflammation and oxidative stress via PI3K/AKT/NF-kB signaling in the granulosa cells of PCOS patients. J Clin Endocrinol Metab 100: 201-211, 2015.

54. Zhao Y, Qiao J, Zhang C, Huang Y and Liu P: Increased WNT5a expression upregulates inflammation via $\mathrm{PI} 3 \mathrm{~K} / \mathrm{AKT} / \mathrm{NF}-\kappa \mathrm{B}$ signaling in the granulosa cells of PCOS patients. Fertility Sterility 102 (Suppl 3): e27-e28, 2014.

55. Xin MA and Wang R: Endometrial lesions mechanisms for insulin to promote PCOS through PI3K/AKT/GSK3 pathways in PCOS patient. Labeled Immunoassays Clin Med 22: 330, 2015.

56. Weickert MO, Hodges P, Tan BK and Randeva HS: Neuroendocrine and endocrine dysfunction in the hyperinsulinemic PCOS patient: The role of metformin. Minerva Endocrinol 37: 25-40, 2012

57. Karar J and Maity A: PI3K/AKT/mTOR pathway in angiogenesis. Front Mol Neurosci 4: 51, 2011 